\title{
Autoevaluación y Emociones en la Formación Inicial de Profesores de Educación Física
}

\author{
Self-Assessment and Emotions in the Initial Education of Physical Education Teachers
}

Autoavaliação e emoções na formação inicial de professores de Educação Física

\author{
Alberto Moreno D. ${ }^{\mathrm{a}}$, Carmen Trigueros $C .^{\mathrm{b}}$ y Enrique Rivera $G .^{\mathrm{b}}$ \\ ${ }^{a}$ Grupo de Estudio Motricidad y Educación. Escuela de Educación Física, Pontificia Universidad Católica de \\ Valparaíso. Correo electrónico: alberto.moreno@ucv.cl. Telf.: (56) (32) 2274383

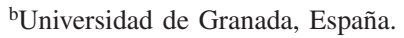

\begin{abstract}
RESUMEN
Estudio cualitativo que analiza las percepciones del alumnado participante en el proyecto "Democratizar el aula universitaria: formar docentes, formar personas" (DAU), llevado a cabo por profesores del Área de Expresión Corporal del Departamento de Didáctica de la Expresión Musical, Plástica y Corporal de la Universidad de Granada, España, en relación a las emociones vividas y experimentadas en el proceso de autoevaluación que dicho proyecto propone. Para ello analizamos los discursos de 49 participantes, hombres y mujeres, con distintos niveles de participación. Se utilizó entrevistas en profundidad y relatos escritos. La información producida, una vez transcrita, se estudió desde el análisis de contenido apoyado en el programa computacional Atlas ti 5.0. Los procedimientos de análisis empleados, en general, se ciñeron a las propuestas de fragmentación y articulación de la "Grounded Theory". Se concluye que la emoción entendida como la aceptación del otro como legítimo otro en la convivencia, ha sido la piedra angular del proceso de autoevaluación vivenciado, pues ha inducido a la implicación activa, responsable y ética en dicho proceso.
\end{abstract}

Palabras clave: formación de profesores, educación física, autoevaluación, emoción.

\begin{abstract}
Qualitative study that analyzes the perceptions of students participating in the project "Democratization of the University class: educating teachers, educating people", carried out by professors in the area of body expression in the Department of musical, plastic and body expression, at the University of Granada, Spain, in relation to the self-assessment process this study proposes. We analyze the discourse of 49 participants, male and female, with different levels of participation. Interviews in depth and written essays were used. There was a content analysis of the generated information (once it was transcribed) with the support of the software Atlas ti 5.0. In general, the analysis procedures were focused on the fragmentation and articulation proposals of the "Grounded theory". The conclusion is that the emotion, understood as the action of accepting one another as legitimate in the coexistence, has been the base of the self-assessment, as it has encouraged an active, responsible, and ethical participation in that assessment.

Key words: teacher education, physical education, self - evaluation, emotion.
\end{abstract}

\section{RESUMO}

Estudo qualitativo que analisa as percepções de alunos participantes no projeto "Democratizar a sala de aula universitária: formar docentes, formar pessoas" (DAU), desenvolvido por professores da Área de Expressão Corporal do Departamento de Didática da Expressão Musical, Plástica e Corporal da Universidade de Granada, Espanha, em relação às emoções vividas e experimentadas no processo da autoavaliação proposta pelo respectivo projeto. Para isso, analisaram-se os discursos de 49 participantes, homens e mulheres, com diferentes níveis de participação. Usaram-se entrevistas profundas e produções textuais. A informação produzida, uma vez transcrita, foi estudada desde a análise de conteúdo apoiada no programa computacional Atlas ti 5.0. Os procedimentos de análise usados, em geral, se vinculam às propostas de fragmentação e articulação da "Grounded Theory". Conclui-se que a emoção, entendida como a aceitação do outro como legítimo outro na convivência, foi a pedra angular do processo de autoavaliação vivenciado porque induziu à implicação ativa, responsável e ética neste processo.

Palavras chave: formação de professores, educação física, autoavaliação, emoção. 


\section{INTRODUCCIÓN}

En el presente trabajo presentamos los resultados de una investigación acerca del proceso de evaluación (autoevaluación) seguido en el proyecto de innovación pedagógica "Democratizar el aula universitaria: formar docentes, formar personas" (DAU) llevado a cabo por profesores del Área de Expresión Corporal del Departamento de Didáctica de la Expresión Musical, Plástica y Corporal de la Universidad de Granada, España.

La democratización de los espacios y tiempos educativos requiere, entre otras cosas, atender a formas de evaluación entendidas como procesos de diálogo, comprensión y mejora (Santos, 1995). El proyecto Pedagógico "DAU" parte de dos ideas claves y cuatro apartados que lo definen. La primera idea tiene relación con entender que la educación no es neutral sino que, por el contrario, tiene una fuerte carga ideológica que condiciona todo proceso formativo. En cuanto a la segunda idea, la educación cobra sentido si es proyectada en la comunidad que la acoge, manteniendo como utopía el logro de transformar la sociedad hacia modelos cada vez más democráticos, de participación, justicia e igualdad de oportunidades.

Los cuatro apartados esenciales que lo definen son: la transdisciplinareidad, la democratización del aula, una metodología colaborativa identificada con los principios socioconstructivistas y los procesos de autoevaluación (De la Torre, Rivera y Trigueros, 2007).

La transdisciplinareidad se proyecta en la fusión de dos materias que comparten tiempos y espacios pedagógicos, metodológicos y de evaluación, con una tercera que al ser optativa, sólo permite compartir, en parte, el espacio y el tiempo pedagógico, pero que respeta la metodología y la evaluación. La democratización del aula se fundamenta en la participación colegiada en la toma de decisiones, el compromiso social y la emancipación de todos los que participan en el proceso (docentes y estudiantes). Se pretende participar de un espacio en situación de libertad e igualdad, donde la tolerancia, la solidaridad, la dignidad, la justicia, la autonomía y la responsabilidad deben ser visibles en lo cotidiano.

En cuanto a la metodología y siendo coherentes con los aspectos anteriores, se orienta a que los estudiantes tengan en su poder decidir sobre su ritmo de aprendizaje, que estos sean conectados con la realidad contextual de la escuela y con los intereses del alumnado; que el docente sea mediador del proceso de aprendizaje, que la crítica, planteada con alternativas para la mejora, sea el motor que facilite el aprendizaje entre iguales. Poner el énfasis en la observación, análisis, discusión y modificación de la realidad educativa. Ello se complementa y enriquece con lecturas, exposiciones y reflexiones críticas. Por último, indicar la importancia y relevancia otorgada al uso constante de las tecnologías de la información y la comunicación.

Una propuesta centrada en la democratización del aula precisa de una evaluación en consecuencia; y si esta debe culminar con la toma de conciencia del proceso vivido por parte del estudiante, quién mejor que él o ella para realizarlo como protagonista apoyado o apoyada por el resto de participantes (docentes y compañeros). Esta es la razón por la apuesta que se hace por la autoevaluación, culminada en un proceso de auto-calificación.

En este contexto, escuchar la voz del alumnado acerca de los procesos evaluativos vividos abre una puerta al análisis sobre la Formación de Profesores de Educación Física (en adelante FPEF) en la Universidad Española, para poder así adaptar ésta a los requerimientos educacionales de la sociedad del conocimiento en que estamos inmersos. Cobra aún más sentido en estos momentos en los que la Universidad Española está viviendo el proceso de convergencia europea que todos conocemos. 


\section{EMOCIONES Y EDUCACIÓN: ALGUNAS NOTAS PRELIMINARES}

Para nadie es desconocida la posición periférica de las emociones en las instituciones educativas formales, independiente del nivel académico al que nos estemos refiriendo, aun admitiendo que los sentimientos y afectos no quedan en la puerta de las instituciones, sino que ingresan con las personas que allí habitan (Santos, 2007). Si bien esta situación es, a lo menos, sorprendente, no es menos cierto que es coherente con el sustrato epistemológico que aún sustenta las instituciones educativas, como es el paradigma cartesiano (Calvo, 2005; Moreno y Calvo, 2010). Un sustento que niega la emoción por sobre la razón y que ni siquiera permite dialogar a ambas realidades (Calvo, 2005).

Este escenario ha originado que las emociones hayan generado escasa atención por parte de la investigación científica en el siglo pasado. Pekrun (2005) identifica esta realidad y señala dos excepciones: estudio de la ansiedad y su relación con la evaluación y el rendimiento académico y el análisis de la relación entre emoción -motivación y éxito- y fracaso académico.

Si bien disponemos de pocas referencias investigativas en torno a la temática de las emociones, no lo es menos que en las dos últimas décadas pareciera surgir un interés al respecto (Casassus, 2006; López Sánchez, 2009). La literatura actual, cuando aborda las emociones en el ámbito educativo y del aprendizaje, suele estar bastante de acuerdo en que las mismas están condicionadas, determinadas y/o relacionadas con respuestas fisiológicas, patrones cognitivos -subjetivos y aspectos de expresión de dichas emociones (González, 2006).

Sea como fuere, lo que la investigación deja bastante claro es que las emociones ya no son algo que le pertenezca únicamente a la herencia animal del ser humano, sino que juegan un papel primordial tanto en los procesos racionales (Damasio, 1999; Goleman, 1995) como en las formas de relacionarnos socialmente (Chovil, 1997; Fridlund, 1997).

Entendemos entonces que los procesos de enseñanza y aprendizaje están condicionados por procesos emocionales que los sustentan y le dan sentido. Es importante recordar la revolución conceptual propuesta por Maturana y Varela (2003) al contradecir la teoría clásica del conocimiento que antepone razón a emoción. Ellos sustentan que es la emoción la que direcciona los procesos racionales, pues están en su base.

Esta perspectiva teórica de las emociones considera que las mismas no se dan en sujetos individuales, sino que tienen que ver con procesos relacionales mediadas por una cultura determinada (Rebollo y otros, 2008). Entendemos, entonces, por emoción, un conjunto de "conductas relacionales" (Maturana, 1999: 221) que emergen en la cotidianeidad del vivir. E identificamos dos grandes emociones, "el amor, como conducta relacional a través de la cual el otro surge como legítimo otro" y la "agresión como el dominio de aquellas conductas a través de las cuales el otro surge negado como legítimo otro en coexistencia con uno mismo" (Maturana, 1999: 221).

Es desde aquí que analizaremos los procesos emocionales en su dimensión narrativa, pues las relaciones significativas que los sujetos establecen con otra personas, lugares y sucesos se expresan en el lenguaje en relación con los otros (Maturana, 2002). 


\section{FORMACIÓN DE PROFESORES DE EDUCACIÓN FÍSICA, PROCESOS AUTOEVALUATIVOS E INTENCIONALIDAD PEDAGÓGICA TRANSFORMADORA}

La Educación Física se ha caracterizado por una falta de análisis ideológico que ayude a analizar los condicionantes que han limitado la puesta en práctica de lo que se entiende por una EF verdaderamente educativa, lo que ha originado que las teorías educativas reproductoras tuvieran tanta presencia en la disciplina (Kirk, 2001, 2004). Es innegable que las prácticas tecnocráticas han tenido y siguen teniendo mucha presencia en el área, pero no es menos cierto que han existido movimientos alternativos que han estudiado, analizado y propuesto alternativas al respecto (Bain, 1993; Fernández, 1997; López y otros, 2007; Rivera, De la Torre y Trigueros, 2009), todas ellas ligadas a perspectivas educativas sociocríticas (Moreno, 2011).

Uno de los aspectos centrales en los que estas propuestas han centrado sus esfuerzos ha sido el tema de la evaluación, en el supuesto de que esta no es una tarea técnica sino que ella impregna toda la lógica pedagógica que los profesores siguen y que, además, condiciona y determina una forma tecnocrática de entender la Formación de Profesores de Educación Física (FPEF) que no atiende a la diferentes problemáticas sociales (Moreno, 2011). Si entendemos que la educación en general y los procesos pedagógicos que en ella se abordan no son neutros sino que están cargados por una fuerte carga ideológica que condiciona todo proceso formativo (Fernández, 1995), también debiéramos comprender que toda acción que se denomine educativa debe intencionar una práctica que contribuya a la "transformación social y cultural centradas en la dignidad y la justicia a través de la democracia" (Rivera y De la Torre, 2005: 94).

Es por ello que la democratización de los espacios y tiempos educativos ha sido un eje articulador en las propuestas sociocríticas en la formación de profesores de EF (López y otros, 2002; Rivera y Otros, 2010). En ellas, la reflexión crítica es un foco central para la construcción social y participativa del conocimiento (Fraile, 2003) lo que requiere la de-construcción de las conceptualizaciones existentes sustentadas en el paradigma tecnocrático de la enseñanza y el aprendizaje en la FPEF. Para ello es necesario configurar un proceso dialógico de construcción de conocimientos negociados y consensuados surgido a partir del trabajo colaborativo de todos los implicados en la acción pedagógica (Fraile, 2006), es decir, en una FPEF centrada en la generación de autonomía, pensamiento crítico y capacidad reflexiva de los estudiantes.

En dicho proceso de diálogo es donde la autoevaluación cobra verdadera importancia, pues permite consensuar colegiadamente, a través de la participación del alumnado en la toma de decisiones, el proceso de formación seguido (López, 2004). Estaríamos pasando, entonces, de una FPEF centrada en el qué y cómo enseñar a una práctica formativa y transformativa, que desarrolla los por qué y para qué hacerlo y que apuesta por el desarrollo de las capacidades morales, ética y políticas de los futuros ciudadanos (Fernández, 2004).

Este trabajo pedagógico en la FPEF requiere de una serie de procedimientos metodológicos que atiendan a la justicia, participación y diálogo en el quehacer profesional en colaboración con los estudiantes y el resto de la comunidad. Muros (2006) señala, para esta práctica emancipadora, que la autoevaluación es una ayuda en la autodeterminación y autorregulación de los estudiantes, a través del reconocimiento de que si en la democracia 
debemos valorar y validar la voz de los ciudadanos, los sistemas de evaluación y los otros procedimientos pedagógicos deben ser coherentes con dicho principio.

Es importante considerar que la puesta en acción de procesos autoevaluativos no está exenta de dificultades prácticas, debido a diversos motivos. Sicilia y Dumitru (2009) destacan la importancia de reconocer tales dificultades y tensiones en el trabajo y desarrollo de la autonomía en el contexto de la FPEF. La primera tensión está relacionada con la creencia, un tanto ingenua, de que ceder a los estudiantes en formación la responsabilidad en la toma de decisiones liberará al profesor del riesgo que conlleva ejercer poder sobre los alumnos. Otra dificultad importante está relacionada con la incapacidad, en muchas ocasiones, de entender que la puesta en práctica de la autoevaluación no se limita, únicamente, a un trabajo educativo anclado en la relación profesor y alumno, sino que se hace indispensable orientar el cambio, no sólo a esa relación, sino al contexto institucional donde dicha relación hace acto de presencia.

En la revisión de la literatura sobre autoevaluación y FPEF nos encontramos con que esta se incluyen en propuestas más amplias de evaluación conceptualizadas como evaluación formativa y participativa. En este contexto la literatura es abundante (Andreu y García, 2006; Anglés y otros, 2006; Biggs, 2005; Boud, 1995 y 1999; Boud y Falchikov, 2006; Gibbs y Simpson, 2009; López 2004, 2008, 2009), aunque en los países de habla hispana es muy escasa la documentación científica que, más allá de describir y valorar cómo se han implementado y aplicado estas dinámicas evaluativas, aporten evidencias que confirmen pertinencia y viabilidad. En coherencia con esto, prácticamente es inexistente las propuestas autoevaluativas que consideren, explícitamente, los aspectos emocionales ligados al diálogo, el trabajo colaborativo-compartido, la autocrítica y todos los factores relacionados con la evaluación formativa-compartida. Haciendo un análisis del libro publicado por la Red Universitaria de Evaluación Formativa y Mejora del Aprendizaje en el EEES (Espacio Europeo de Educación Superior) en el año 2009, "Evaluación Formativa y Compartida en Educación Superior" (López, 2009), es interesante señalar que solo una de las experiencias narradas (Chivite y Romero, 2009) hace mención explícita a la problemática emocional en los procesos de evaluación. Ello debido, probablemente, a las dificultades propias de atender a procesos tan complejos, abstractos y difíciles de evaluar como es la trama emocional presente en cualquier proceso de enseñanza y de aprendizaje. Es por esta última razón que consideramos relevante lo que presentamos en nuestra investigación, pues podría ser el inicio de una línea de trabajo que invitara a la reflexión sobre esta problemática educacional en el contexto de la FPEF.

\section{DISEÑO METODOLÓGICO}

El trabajo es un estudio fenomenológico y estamos dentro de lo que se ha dado en llamar, en investigación social, el paradigma interpretativo. Para su desarrollo nos apoyaremos en la metodología cualitativa, ya que el estudio posee un carácter descriptivo, relacional e interpretativo (Fraile y Vizcarra, 2009).

¿Cuál ha sido el detonante del mismo? Las preguntas surgen a partir del elemento que se identifica como el más controvertido o polémico de toda la propuesta: dejar en manos del estudiante el poder de la evaluación. Desde aquí se plantean inicialmente las siguientes preguntas de investigación: 
- ¿Cómo valoran el proceso de autoevaluación seguido?

- ¿Cuáles son las fortalezas y debilidades que detectan en él?

- ¿Cuál es el significado que otorgan a tener que responsabilizarse de su propio proceso de aprendizaje mediante la participación activa en la toma de decisiones durante el proceso de autoevaluación?

- ¿Qué papel han jugado las emociones en el proceso de autoevaluación experimentado durante el proyecto Democratizar el Aula Universitaria (DAU)?

Estas preguntas previas nos ayudan a identificar el objeto de la investigación: visibilizar las creencias y teorías implícitas de los estudiantes participantes en el proyecto, concretando el mismo en un objetivo general de investigación:

- Describir, analizar y comprender las percepciones de los estudiantes en relación el rol jugado por las emociones en el desarrollo de la propuesta autoevaluativa durante el proyecto.

Los participantes en la investigación son estudiantes de la especialidad de Magisterio de Educación Física de primer y segundo año de una Facultad de Ciencias de la Educación donde se implementa el proyecto pedagógico.

El total de participantes que entregaron relatos escritos ascendió a 38, mientras que 11 estudiantes fueron entrevistados. La selección de los mismos para las entrevistas se realizó a partir de dos criterios básicos: asistencia a clase y calificación obtenida, pues era importante considerar el nivel de implicación y conocimiento vivenciado del proceso de autoevaluación para poder opinar acerca del mismo.

\subsection{INSTRUMENTOS}

- Relato escrito en donde los estudiantes evalúan el proyecto "DAU" y en donde se evalúa, en concreto, el proceso de autoevaluación seguido. El relato utilizado consiste en una narración escrita en donde se da cuenta de la experiencia vivida durante el proyecto en el que han participado y el proceso de autoevaluación vivenciado. Cada historia como estudiante puede tener diferentes principios. "La propuesta es que cada uno elabore su propio relato de acuerdo a su propia experiencia y a la forma como cada uno percibe su propia historia" (Martínez, 2006: s/p).

- Entrevistas en profundidad al alumnado para penetrar en la evaluación que ellos hacen del proceso autoevaluativo seguido en el proyecto y los significados que ha tenido el mismo para ellos. La entrevista como técnica tiene como propósito que el investigador se sitúe en el lugar del entrevistado, suponiendo que la realidad de los otros es significativa, cognoscible y explícita (Flick, 2004; Goetz y Lecompte, 1988). Teniendo en consideración lo anterior, por entrevistas cualitativas en profundidad se debe entender los reiterados encuentros cara a cara entre el investigador y los informantes, encuentros dirigidos hacia la comprensión de las perspectivas que tienen los informantes respecto de sus vidas, experiencias o situaciones, tal como las expresan sus propias palabras (Flick, 2004; Taylor y Bogdan, 1984).

\subsection{ANÁLISIS REALIZADOS}

Toda la información recabada a lo largo del trabajo de campo fue transcrita y sometida a un primer análisis de discurso siguiendo las pautas marcadas desde la "Grounded 
Theory" (Glaser y Strauss, 1967; Strauss y Corbin, 2002, Valles, 2003) y apoyada en su ejecución por el programa computacional Atlas Ti 5.0. El primer paso fue hacer visibles las teorías sustantivas de los participantes; para ello se realiza una codificación abierta que permite hacer emerger las primeras categorías. Posteriormente, se procede a la elaboración de un primer modelo exploratorio mediante la agrupación de las categorías emergentes en familias de categorías; se realiza una codificación selectiva que permite pasar de la descripción a la interpretación y confrontación de las teorías sustantivas con las formales.

Una vez realizada la primera codificación (creación de nodos libres) y construido un primer árbol de categorías (nodos ramificados), procedimos a la creación de un mapa conceptual que nos permitiera profundizar, aún más, en el análisis, descripción e interpretación del discurso presente en las entrevistas y relatos escritos. El mapa conceptual es el siguiente:

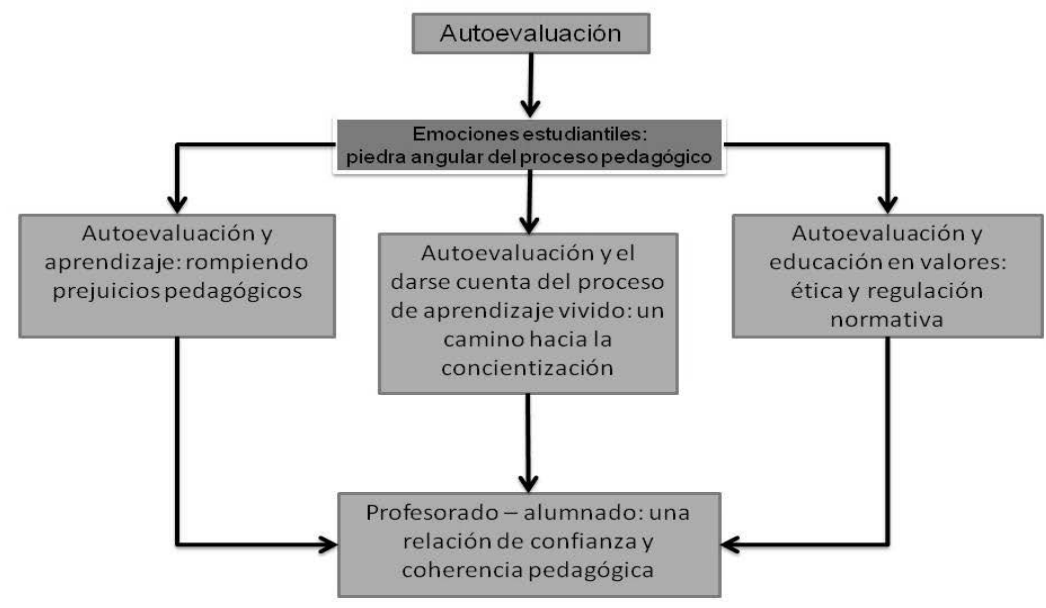

Es relevante, en una investigación de corte cualitativo como la que se describe, dar cuenta de los criterios éticos que nos guiaron en nuestro trabajo metodológico. Hammersly y Atkinson (1994) enfatizan la importancia de la ética en los procesos de investigación cualitativa, atendiendo principalmente a los diferentes comportamientos de los investigadores y sus consecuencias para las personas que forman parte de los estudios. Los criterios seguidos fueron: consentimiento informado, privacidad, perjuicio y consecuencias para investigaciones futuras.

\section{LAS EMOCIONES COMO PIEDRA ANGULAR DEL PROCESO PEDAGÓGICO DE LA AUTOEVALUACIÓN: RESULTADOS EMERGENTES}

\footnotetext{
"Es una forma muy humana, creo yo. Las relaciones personales se trabajan mucho en la autoevaluación ... Entonces una persona levantó la mano para decir que se merecía más nota. Yo creo que es lo mejor que le puede pasar a una persona... creo que se merece más por tal tal y tal..., pues para esa persona deber ser excepcional" (Doc. 1: 069).
} 
Comenzamos con esta cita de uno de los sujetos de investigación porque, de alguna manera, refleja la piedra angular del proceso de autoevaluación experimentado durante el proyecto "DAU". Dicha piedra angular y que, sin duda, ha influido de forma notoria en las otras características del proceso pedagógico, son las emociones vividas por el estudiantado durante la autoevaluación. Dichas emociones han sido principalmente positivas, es decir, de aceptación y no negativas o de negación.

El alumnado siente que esa relación de 'aceptación del otro como legítimo otro en la convivencia' (Maturana, 2002) entre los diferentes estudiantes y, sobre todo, entre discentes y docentes, los condujo no sólo a aceptar la propuesta "DAU" y el proceso de autoevaluación que ella propone, sino también, y esto nos parece lo más relevante, a implicarse activa, responsable y éticamente en ella.

“... es otro de los valores que esta asignatura te da, porque la responsabilidad de llevar el trabajo al día, ser responsable con respecto a lo que te quieras proponer, con tus compañeros, todo... Es una asignatura que te hace ser importante porque tienes que ser responsable con todo..." (Doc. 7: 058).

Cuando hablamos de emoción hacemos referencia al proceso de 'aceptación del otro como legítimo otro en la convivencia', tal y como lo plantea Maturana (2002), entendiendo que el proceso emocional es lo que constituye al ser como 'ser humano' y que en función de dicha emoción emergen diferentes procesos relacionales y racionales.

Como ya comentamos más arriba, esta relación entre todos los implicados en el proceso autoevaluativo se ha convertido en la piedra angular del proceso pedagógico, pues en el proyecto "DAU" y el proceso de autoevaluación seguido, se han diferenciado claramente dos momentos claves de la tarea educativa: la formación humana y la capacitación.

Entendemos que la "formación humana tiene que ver con el desarrollo del joven como persona capaz de ser co-creadora con otros de un espacio humano de convivencia social deseable" (Maturana, 2002: 15). En función de esta aclaración, podemos decir que nuestro estudio nos muestra como el alumnado pone énfasis en estos aspectos emocionales que les han permitido co-crear juntos.

"Por eso te digo que, cuando tu trabajas con la práctica como lo hemos hecho todos en conjunto, las emociones no se pueden hacer desaparecer, porque las emociones surgen con las relaciones y estamos constantemente relacionados, pero cuando estás en tu puesto y tu profesor te explica algo de la forma tradicional, las relaciones son realmente nulas. ¿Qué vas a hablar con él? Da lata decirle que tienes hambre, ¿cuándo acabas para ir a desayunar? ¿Me entiendes lo que te quiero decir? No es una situación significativa, apuntas ahí y ya está. De esta forma hemos hecho clases teóricas varias veces. Esas que hemos hecho son realmente necesarias, pero con esas es suficiente, porque realmente como aprendes es haciendo" (Doc. 1: 066).

El alumnado deja constancia de la importancia del contexto real de aprendizaje y enseñanza, pues la significatividad de la realidad educativa en la que más tarde se implicarán es donde aparecen las ambigüedades, contradicciones y paradojas propias de la vida, aspectos estos solapados en prácticas de formación del profesorado inspiradas en el paradigma tecnocrático, que simplifica tanto los procesos formativos que termina haciendo desaparecer la complejidad y riqueza propia de los mismos.

La autoevaluación permite vivir la complejidad propia de los procesos educativos y no esconde todas las contradicciones a las que hacíamos referencia. 
"De qué sirve obtener cantidades prescritas de información sobre geografía e historia, de qué sirve alcanzar la capacidad de leer y escribir, si en el proceso el individuo pierde su alma; si pierde su apreciación de las cosas que valen la pena, de los valores a los que estas cosas hacen referencia; si pierde el deseo de aplicar lo que ha aprendido y, sobre todo, la capacidad para extraer el significado de las experiencias que tenga en el futuro" (Dewey, 1995: 49).

Para que ello ocurra hay dos aspectos principales experimentados durante el proceso de autoevaluación y que tienen relación con la libertad para tomar decisiones de forma autónoma y la libertad como el aspecto que hace emerger un espacio de convivencia agradable y fructífera.

"Es otra manera de trabajar más relajadamente, no tienes esa tensión de decir tengo que estar trabajando para una buena nota y pasar el examen y si no paso el examen soy suspendido ¿no? Aquí vas viendo tu aprendizaje hasta donde tú quieres... Estás dentro de unas normas pero..., hacer e implicarte en lo que verdaderamente quieres aprender es lo realmente positivo" (Doc. 4: 25).

Los espacios y tiempos de confianza generados durante el proceso de autoevaluación, es lo que les ha permitido, según ellos mismos, un aprendizaje más consistente y duradero en el tiempo.

"Para mí hay confianza al hacer las cosas, no es lo mismo estudiarte o aprenderte algo por la fuerza. En este caso una lectura la ves tranquilamente y sabes que si la has leído, tú misma te vas a poner esa nota, no leértela con la presión de que me van a hacer tales preguntas tal día. Yo creo que el aprendizaje es mucho mejor, estás leyendo más tranquilamente que si estás intentando memorizarlo porque luego va a venir otro a ponerte una nota" (Doc. 11: 008).

Quizás esta característica propia del proceso de autoevaluación, las emociones, sea una de las particularidades menos valoradas en los sistemas de formación de profesores más tradicionales. De ahí el poco éxito de los mismos, pues si nos alejamos de las emociones, si las negamos, estamos negando al sujeto que aprende y si lo negamos nunca este se implicará en aquello que queremos forme parte de su formación académica y personal.

Por lo comentando hasta el momento es que decidimos comenzar nuestro análisis de resultados enfatizando la importancia de las emociones en la construcción del aprendizaje del estudiantado en formación durante el proceso de autoevaluación.

“... con los sentimientos, con las emociones, con las sensaciones, como cuando tú trabajas con la práctica, cuando aprendes de la práctica con las interacciones de las personas... ahí surgen emociones. Eso es inevitable, surgen sensaciones. Eso es significativo porque tú te acuerdas de las emociones y las relacionas con los aprendizajes" (Doc. 1: 057).

Las relaciones humanas de respeto, solidaridad, colaboración y aceptación de todos los implicados durante el proceso, permiten una generación de conocimientos sobre la base de la autonomía individual, la autorresponsabilidad y el respeto con los otros.

Para el alumnado, el proceso de autoevaluación ha permitido generar instancia de aprendizaje adecuadas a la realidad social y pedagógica que se vive hoy en las escuelas, pues no se ha artificializado el proceso de formación de profesores creyendo que la consecución de un buen profesional pasa por la relación jerárquica entre alumno y profesor, en donde el primero sabe, conoce, comprende y el segundo debe estudiar pasivamente para generarse esos mismos conocimientos que el profesorado tiene. Las emociones vividas durante el proceso de autoevaluación, como vemos en la cita anterior, es lo que ha permitido hacer mucho más significativo el proceso. 
Las emociones, según Goleman (1995), son un impulso para la acción y la acción es un contexto de generación de nuevas emociones. Nos conducen a actuar en alguna dirección. Es decir, dependiendo de la emoción que haya surgido actuaremos racional y corporalmente hacia un camino determinado. Esta es la principal importancia de las emociones en el proceso de autoevaluación que estamos estudiando.

Podríamos decir que nosotros, los profesores y alumnos, tenemos dos mentes, una que piensa y otra que se emociona. Para Maturana (1999), lo que hace y piensa el ser humano está condicionado por la mente que se emociona, pues ella determina el camino a seguir en la reflexión. En los proyectos de innovación educativa en donde podemos enmarcar la propuesta "DAU" y, por tanto, el proceso de autoevaluación seguido, se da especial relevancia a la importancia de las emociones de todos los implicados en el mismo, ya que conducirán a la verdadera reflexión que genera aprendizaje.

"El profesorado, y el alumnado, también poseen una dimensión emocional que actúa de filtro en la interacción comunicativa de las clases y también actúa de puente entre la estructura y la cultura escolar, por una parte, y la enseñanza de las clases por otra" (Devís y Jimeno: 2001: 78).

Desgraciadamente, estos procesos emocionales son escondidos, soslayados e incluso criticados en muchas propuestas educacionales que se autoproclaman como innovadoras. Los mismos estudiantes entrevistados comparan los procesos de evaluación tradicional y el proceso de autoevaluación en el que han estado implicados, atendiendo a este criterio emocional del que estamos hablando.

“Considero que con esta forma de evaluar la gente se implica más... se aprende más, porque no todos dan examen y tú sabes que al final todos leen en los últimos días y hay gente que tiene capacidad para aprender o para memorizar y aprueba y hay gente que no. Considero que en la facultad cuando un profesor te dice esto es autoevaluación y vamos a trabajar mucho y vamos a tener que estar muy metidos en la asignatura, y el hecho de yo dedicarle tiempo a la semana, 4 ó 5 horas a la asignatura, independiente de lo que te cuesta hacer la lectura, pues por eso yo me tiro más por esa forma, porque pienso que se aprende más sin el miedo al examen final, cuando estás agobiado por dar un examen...” (Doc. 10: 088).

La posibilidad de experimentar los procesos de evaluación no como la instancia última en donde verificar los aprendizajes alcanzados, sino como una instancia de aprendizaje, es la que lleva a este proceso autoevaluativo a disponer espacios y tiempos pedagógicos en donde compartir, con otros y otras, juicios de valor personales y grupales, discutirlos, reflexionarlos, criticarlos y seguir construyendo así el saber pedagógico. Esta construcción no está basada, según hemos podido observar en nuestra investigación, en esa instancia en donde los alumnos y alumnas son clasificados por otros en un lugar que represente más o menos conocimientos, sino que, por el contrario, la construcción se centra en el poder decirles a otros y otras qué ha aprendido cada uno, qué ha sentido, cómo ha influido en cada uno esas emociones y cómo estas han dirigido el aprendizaje en una dirección determinada, una dirección que el proyecto marca como la justicia social, la solidaridad, el respeto y la aceptación del otro.

La coherencia entre el proyecto "DAU" y el proceso de autoevaluación queda evidenciada en la siguiente cita:

"A lo mejor me hubiera puesto un punto más, porque al no haber hecho un seguimiento mío, tampoco puedo yo tirarme más, después, sin embargo, hay muchas asignaturas que pienso que tienen cosas muy comunes con este proyecto y después das examen, es eso lo que te 
molesta, es eso lo que te duele. Si me estás exigiendo lo mismo que aquí, encima estás viendo lo que hice, si me mandas a examen, lo que estás viendo que todo el proyecto, al proceso le quitas importancia y al quitarle importancia, lo que estás haciendo es que perdamos interés, porque simplemente con ese tema de autoevaluación por lo menos los aprendizajes son más significativos, desde luego más duraderos, por supuesto. Yo creo que es lo más importante, es mucho más duradero, creo que de lo de aquí lo que nunca más se me va a olvidar es cómo organizar una clase, cómo distribuir una clase, esos son ya dos hechos, porque ya los estoy encontrando en .... [nombre de asignatura] que era lo que argumentaba el profesor, yo mirando al compañero que también ha hecho este proceso, es que hemos visto de todo" (P2: 125).

\section{REFLEXIONES ÚLTIMAS A MODO DE CONCLUSIONES}

La emoción, entendida como la aceptación del otro como legítimo otro en la convivencia, ha sido la piedra angular del proceso de autoevaluación vivenciado, pues ha inducido a la implicación activa, responsable y ética en dicho proceso.

Dicha emoción ha permitido, según los estudiantes, la co-creación de saberes pedagógicos contextualizados en la realidad escolar en la que ellos, posteriormente, desarrollarán su labor profesional, pues dicho contexto autoevaluativo ha sido un espacio de contradicciones, ambigüedades y paradojas propias de la complejidad educativa y escolar.

Dicha complejidad autoevaluativa ha sido vivida y experimentada gracias a la libertad que los estudiantes han tenido para tomar decisiones de forma autónoma.

La confianza generada durante el proceso de autoevaluación les ha permitido a los estudiantes un aprendizaje más consistente y duradero en el tiempo.

\section{REFERENCIAS BIBLIOGRÁFICAS}

Andreu, Ma . A. y García, M. (2006). Evaluación, coevaluación y autoevaluación del trabajo en grupo en la lectura de mapas topográficos. En F. Watts y A. García (Edit), La evaluación compartida: investigación multidisciplinar (pp. 69-89). Valencia: UPV.

Anglés, M. et al. (2006). Evaluación continua y formativa en un curso universitario de química. En F. Watts y A. García (Edit), La evaluación compartida: investigación multidisciplinar (pp. 165-197). Valencia: UPV.

Bain, L. L. (1993). Ethical issues in teaching. Quest, n. 45, 69-77.

Biggs, J. B. (2005). Calidad del aprendizaje universitario. Madrid: Narcea.

Boud, D. (1995) (Ed). Enhancing learning throuhg self assesment. London: Routledge Falmer.

Boud, D. (1999). Avoiding the traps: seeking good practice in the use of self assessment and reflection in profesional courses. Social Work Education, n. 18, 2, 121-132.

Boud, D. y Falchikov, N. (2006). Aligning assessment with long-term learning. Assesment and Evaluation in Higher Education, vol. 31, n. 4, 399-413.

Calvo, C. (2005). Complejidad, caos y educación informal. En A. Arellano (Coord.), La educación en tiempos débiles e inciertos (pp. 115-136). Barcelona: Editorial Anthropos.

Casassus, J. (2006). La educación del ser emocional. Santiago de Chile: Cuarto Propio.

Chivite, M. y Romero, Ma . (2009). Evaluación de competencias con fuerte componente emocional y afectivo: un sistema de evaluación formativo y continuo. En V. López (Coord), Evaluación formativa y compartida en educación superior. Propuestas, técnica, instrumentos y experiencias (pp. 204-208). Madrid: Narcea. 
Chovil, N. (1997). Facing others: A social communicative on facial display. En Russell, J and Fernández-Dols, J.M (Edit.), The Psychology of Facial Expression. Cambridge: Cambridge University Press.

Damasio, A. (1999). El error de Descartes. Santiago de Chile: Andrés Bello.

De la Torre, E.; Rivera, E. y Trigueros, C. (2007). Creencias y concepciones de la educación física en evolución: el caso de la formación del profesorado de educación física en la educación primaria. Revista Tándem. Didáctica de la Educación Física, n. 23, 50-56.

Devís, J. y Jimeno, L. (2001). Emociones, profesorado e innovación en la educación física: la revisión de un estudio de casos. En J. Devís (Coord.), La Educación Física, el deporte y la salud en el siglo XXI. Alicante: Editorial Marfil.

Dewey, J. (1995). Democracia y educación. Una introducción a la filosofía de la educación. Madrid: Morata.

Fernández, J. M. (2004). Recuperando el valor ético-político de la Pedagogía: las diferencias entre la Pedagogía y la Didáctica. En Fraile, A (Coord.), Didáctica de la Educación Física. Una perspectiva crítica y transversal (pp. 315-330). Madrid: Biblioteca Nueva.

Fernández, J. M. (1995). Reclaiming physical education in higher education through critical pedagogy. Quest, vol. 47, n. 1, 91-114.

Fernández, J. M. (1997). La investigación en la educación física Española: un índice para el futuro. I Encuentro de Profesores Universitarios de Didáctica de la educación Físico-deportiva. Málaga: Instituto Andaluz del Deporte.

Flick, U. (2004). Introducción a la investigación cualitativa. Madrid: Editorial Morata.

Fraile, A. (2003). Un cambio democrático en las aulas universitarias: una experiencia en la formación del profesorado de educación física. Contextos Educativos. Revista de Educación, $n$. 6-7, 213-234.

Fraile, A. (2006). Cambios en el aula universitaria ante los nuevos retos europeos. Revista Tándem. Didáctica de la Educación Física, 20, 57-72.

Fraile, A. y Vizcarra, T. (2009). La investigación naturalista e interpretativa desde la actividad física y el deporte. Revista de Psicodidáctica, vol. 14, n. 1, 119-132.

Fridlund, A. (1997). The new ethology of human facial expression. En Russell, J \& Fernández-Dols, J. M. (Edit.). The Psychology of Facial Expression. Cambridge: Cambridge University Press

Gibbs, G., y Simpson, C. (2009). Condicions per a una avaluació continuada que afavoreixi l'aprenentatge. Barcelona: Octaedro ICE. Universitat de Barcelona.

Glasser, B. y Strauss, A. (1967). The discovery of grounded theory. Strategies for qualitative research. Chicago: Aldine Publishing Company.

Goetz, J. P. y Lecompte, M. D. (1988). Etnografía y diseño cualitativo en investigación educativa. Madrid: Morata.

Goleman, D. (1995). Inteligencia emocional. Barcelona: Kairós.

González, G. (2006). Aspectos Psicológicos y Neurales en el Aprendizaje del Reconocimiento de Emociones. Revista Chilena de Neuopsicología, vol. 1, n. 1, 21-28.

Hammersly, M. y Atkinson, P. (1994). Etnografía. Métodos de Investigación. Barcelona: Paidós.

Kirk, D. (2001). Fundamentos para una pedagogía crítica en la formación del profesorado de Educación Física. En Devís, J. (Coord.), La educación física, el deporte y la salud en el siglo $X X I$. Alicante: Editorial Marfil.

Kirk, D. (2004).Towards a critical history of the body, identity and health: Corporal power and school practice. En Evans, J.; Davies, B. y Wright, J. (Coords.), Body knowledge and control: Studies in the sociology of physical education and health (pp. 52-67). Londres: Routledge.

López Sánchez, F. (2009). Las emociones en educación. Madrid: Morata.

López, V. M. (2004). La participación del alumnado en los procesos evaluativos: la autoevaluación y la evaluación compartida en Educación Física. En Fraile, A. Antonio (Coord.), Didáctica de la Educación Física. Una perspectiva crítica y transversal. Madrid: Editorial Biblioteca Nueva. 
López, V. M. (2008). Desarrollando sistemas de evaluación formativa y compartida en la docencia universitaria. Análisis de resultados de su puesta en práctica en la formación inicial del profesorado. European Journal of Teacher Education, vol. 31, n. 3, 293-311.

López, V. M. (Coord.). (2009). Evaluación formativa y compartida en educación superior. Propuestas, técnicas, instrumentos y experiencias. Madrid: Narcea.

López, V. M. et al. (2007). La atención a la diversidad en el área de Educación Física. La integración del alumnado con necesidades educativas específicas, especialmente el alumnado inmigrante y de minorías étnicas. Revista Educación Física y Deportes, vol. 11, n. 106. En línea; disponible en http://www.efdeportes.com, [consulta 2010, 12 de abril]

López, V. M. et al. (2002) Algunas reflexiones sobre educación física y pedagogía crítica. Retos. Nuevas tendencias en educación, deportes y recreación, $n$. 2, 30-35.

Martínez, J. B. (2006). Proyecto de investigación presentado para optar al cuerpo de Catedrático de Universidad. Material multicopiado no publicado.

Maturana, H. (1999). Transformación en la convivencia. Santiago de Chile: Dolmen Ediciones.

Maturana, H. (2002). Formación humana y capacitación. Santiago de Chile: Dolmen Ediciones.

Maturana, H. y Varela, F. (2003). El árbol del conocimiento: las bases biológicas del entendimiento humano. Buenos Aires: Lumen.

Moreno, A. (2011). Percepciones del profesorado universitario en relación a la función de transformación de la educación física como asignatura de curriculum escolar: el caso de Chile. Tesis Doctoral Inédita. Granada: Universidad de Granada

Moreno, A. y Calvo, C. (2010). Etnoeducación, Educación Física y Escuela: transitando desde la educación informal a la escuela autoorganizada. Ágora para la Educación Física y el Deporte, vol. 12, n. 2, 131-150.

Muros, B. (2006). La puesta en práctica de la pedagogía critica. Estategias metodológicas crítica. Tándem: Didáctica de la Educación Física, vol. 20, 33-44.

Pekrun, R. (2005). Progress and open problems in educational emotion research. Learning and Instruction, n. 15, 497-506.

Rebollo, $\mathrm{M}^{\mathrm{a}}$. A. et al. (2008). Las emociones en el aprendizaje online. Relieve, v. 14, n. 1, 1-23. http://www.uv.es/RELIEVE/v14n1/RELIEVEv14n1_2.htm [consulta 2011, 29 junio]

Rivera, E.; De la Torre, E. y Trigueros, C. (2009). Formar docentes, formar personas: la formación inicial del profesorado desde una propuesta sociocrítica. Conferencia presentada al I Ciclo sobre Complejidad y Modelo Pedagógico. (http://www.tendencias21.net/ciclo/ Un-centropara-formacion-en-la-complejidad-de-educadores-y-docentes-dara-continuidad-al-Ciclo_a68. html [consulta 2009, 27 de abril]

Rivera, E.; De la Torre, E. (2005). Democratizar el aula universitaria: una propuesta alternativa de formación inicial universitaria desde la participación del alumnado. Investigación en la Escuela, n. 57, 85-95.

Rivera, E. et al. (2010). Formar docentes, formar personas: una experiencia transdisciplinar para democratizar el aula universitaria. En L'Activitat del Docent: Intervenció, Innovació, Investigació (pp. 1-8). Barcelona: CiDd: II Congrés Internaiconal de Didáctiques.

Santos, M. A. (1995). La evaluación: un proceso de diálogo, comprensión y mejora. Málaga: Ediciones Aljibe.

Sicilia, A. y Dumitru, D. (2009). La formación de sujetos autónomos a través de procesos de autoevaluación y autocalificación. Tensiones y Conflictos. En V Congreso internacional de evaluación formativa en docencia universitaria: La Evaluación Formativa en el Proceso de Convergencia hacia el Espacio Europeo De Educación Superior, S/P.

Strauss, A. y Corbin, J. (2002) Bases de la investigación cualitativa. Técnicas y procedimientos para desarrollar la teoría fundamentada. Colombia: Universidad de Antioquia.

Taylor, S. y Bogdan R. (1984). Introducción a los métodos cualitativos de investigación. Barcelona: Paidós.

Valles, M. (2003). Técnicas Cualitativas de Investigación Social. Reflexión metodológica y práctica profesional. Madrid: Síntesis. 
